\title{
Measurements of the Apparent Solar Diameter with Astrolabe at Observatório Nacional-1997/2000
}

\author{
A. H. Andrei, E. Reis Neto, J. L. Penna, W. G. de Almeida, \\ V. A. d'Ávila, \\ Observatório Nacional, R. Gal. José Cristino 77, RJ, Brazil
}

\author{
S. P. Puliaev and E. G. Jilinski
}

Observatório Nacional, Brazil and Pulkovo Observatory, Russia

\begin{abstract}
The metrological qualities render the modified CCD solar astrolabe a very reliable instrument for the difficult conditions of solar diameter measurements. At O.N. an average value of 959".04 $\pm 0 " .01$ is found, for $\lambda=563.5 \mathrm{~nm}$, and an effective bandpass of $168 \mathrm{~nm}$. To improve the signal to noise ratio, the images are treated for flat field. This includes distortions brought about by the COHU 4710 camera, the astrolabe optics, and by the system of filters that cut down the incoming solar light. The extensive series observed (over 12000 independent measures) is examined, using a CLEAN periodogram algorithm. The outcome shows periods reconcilable with the solar activity and geometry of observation.
\end{abstract}

\section{Introduction}

Investigations about the variability of the solar diameter give important information about the photosphere, as well as constraints on the physics of the solar interior. Presently, the PICARD and SPHERIS satellites are to be launched to measure the solar diameter and constant. On ground observations with DORAYSOL (Damé et al., 1999) will be made at the same time, in order to keep track with the lengthy series of astrolabe results, as well as to follow up the variations found.

At the Observatório Nacional (ON) $\left(\phi=-22^{\circ} 53^{\prime} 42.50^{\prime \prime}, \lambda=+2^{h} 52^{m} 53.479^{s}\right.$, $\mathrm{h}=33 \mathrm{~m}$ ) the observations started in 1997 January, initially to east transits only (Jilinski et al., 1998). The observations are carried out with a modified astrolabe, equipped with a frontal prism of variable angle, which enables to operate all year round. Typically, 30 measurements are made per day, half of them on each side of the meridian transit (Puliaev et al., 2000). In this work, to obtain evenness between east and west observations, only those from 1998 January on are retained, to a total of 10136 determinations of the apparent semi-diameter (4815 eastern's and 5319 western's). The observational wavelength centers at $\lambda$ $=563.5 \mathrm{~nm}$, to an effective bandpass of $168 \mathrm{~nm}$. 


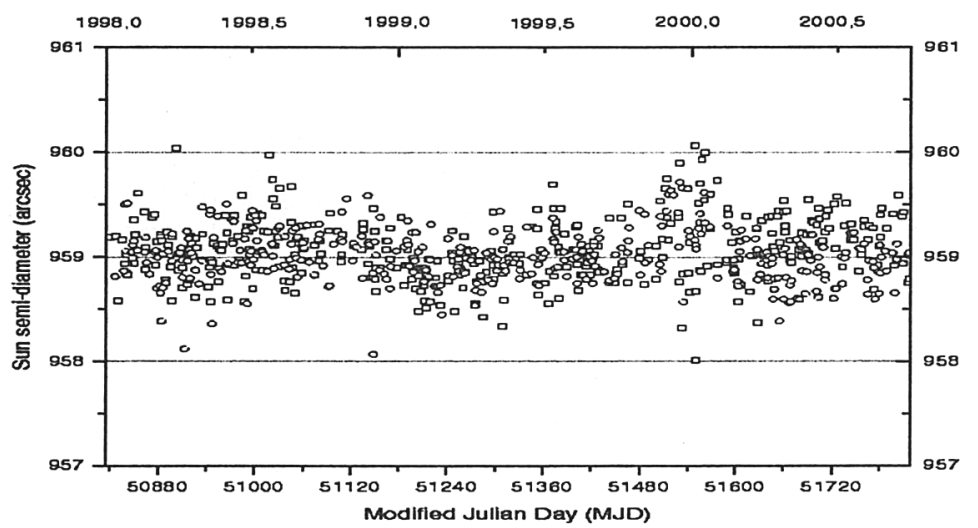

Figure 1. Solar semi-diameter measurements obtained at ON. Each point represents the average of an east (circles) or west (squares) observations session. Errors bars were omitted for clarity, being of 0 ".2 the typical r.m.s. errors associated to the points.

\section{Time Series}

Variations on the solar diameter, as limited by the inflection point of the center to limb darkening function, has been reckoned by several authors (see Ribes et al., 1991). The ON semi-diameter series, from 1998 January up to 2000 September is presented in Figure 1. It contains 369 east observation days and 380 west observation days. The average semi-diameter is $959 " .04 \pm 0$ ".01, to a standard deviation of 0 ".28. The eastern's points average is larger than the western's by 0 ". 07 , which is non-significant regarding the standard deviation.

Longer series analyzed by other authors (Laclare et al., 1996; Rozelot, 1998) point out to periodicities on the variation of the semi diameter, commensurable with the solar cycle. In the present case, the series is of high density, having on average 13 observations per session (either a.m. or p.m.) and 0.75 sessions per day (Jilinski et al., 1999). Such density allows to investigate short scale periodicities. Figure 2 presents the periodograms for the series, separating east and west sessions. A CLEAN algorithm was used (Jablonski, 1991) and the plots are normalized.

Both periodograms show similar features:

a) periods between 10 and 16 days, with maximum amplitude of $0 " .048$. These are commensurable with the short scale solar irradiance variations, although it could be an artifact of observational conditions fluctuation.

b) periods between 20 and 37 days, with maximum amplitude of 0 ".035. Since the observations concentrate around the solar equator, this group is probably tied up to the main rotational period of the Sun.

c) periods between 41 and 78 days, with maximum amplitude of 0 ".037. These periods are suggestive of the sunspots life characteristic time.

d) periods between 129 and 283 days, with maximum amplitude of 0 ".068. These periods are suggestive of either a seasonal or a zenith distance dependence. 

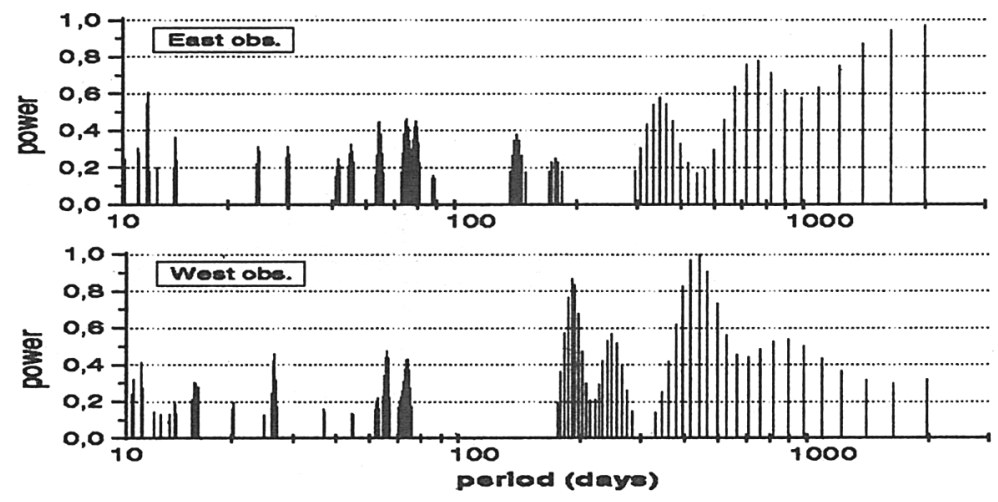

Figure 2. Normalized periodograms for the east and west series of semi-diameter determinations. Periods shorter than 10 days and longer than 2000 days are removed. Only terms with amplitude larger than 0 ". 01 are retained.

e) periods between 295 and 417 days, with maximum amplitude of 0 ".075. They can be associated to quasi-annual variations, whose origin could be either observational or linked to the geometry of the measurements.

f) long periods, from 440 days on, with generally increasing amplitude, up to 0 ".077. They indicate the signature of a growing trend associated to the incoming solar maximum.

\section{Flat Field Correction}

To improve on the signal to noise ratio, a further procedure is being added to the images treatment, in order to account for the different spatial sensibility of the ensemble of instrumental optics and CCD chip (mainly the later). At start, several flat fields are taken over the solar disk. Next, using the IMSURFIT IRAF routine, a smoothing filter, consisting of a second degree bi-dimensional polynomial adjustment, is applied on the flat fields. This minimizes transitory, local fluctuations and the fitted average flat field mask is determined. Still by means of IRAF routines, it is applied on each of the 92 frames gathered for every individual measurement. Following this step, the standard reduction routines (Sinceac, 1998) are used. First tests suggest an accuracy improvement up to 0 ". 057 , and show a 0 ".27 fall on the daily averages standard deviation, when the brightness of the direct and reflected images are similar. Trial flat fields taken on the daylight sky show the count distribution much similar to those taken on the solar disk, although to a much poorer signal to noise ratio. Since all frames are taken with constant integration time $\left(0^{s} .02\right)$, this indicates that dark current effects are in practice dealt with by the flat field mask.

An example of the flat field mask and of its application on the gathered images is shown in Figure 3. The flat field rends less noisy the parables fit to the solar limb. Thus, the straight lines that describe the movement of the parables vertices are better adjusted, and finally a higher accuracy on the instant of touching of the direct and reflected images is achieved. 


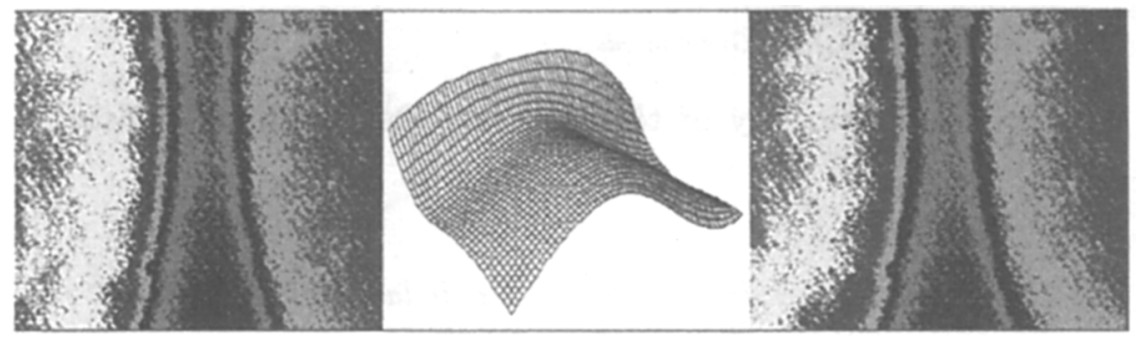

Figure 3. On the left an image before the application of the flat field. On the right, its corresponding after the correction. On the middle the applied flat field mask.

\section{Conclusions}

From the series here analyzed a constant value, or at least only variations smaller than 0".1, cannot be discarded. However, well defined groups of alike periodicities appear when the a.m. and p.m. sessions of observation are treated independently. The main periodicities can be reconciled with the main solar rotation period, with the sunspots life characteristic time, plus long periods that are interpreted as resulting from the approaching of the maximum of the 11 years solar cycle. Periods suggestive of seasonal, observational or linked to the geometry of the observation also appear.

The application of a flat field mask taken on the solar disk upon the acquired images, resulted on a significant gain for the precision of the results.

The ON program is done in collaboration with the CERGA/OCA one, and a second generation instrument is currently being developed. In this way, complementary observations are taken from the two hemispheres, under completely different atmospheric conditions, yet using the same methods.

\section{References}

Damé, L., Hersé, M., Thuillier, G., Appourchaux, T., Crommelynck, D., Dewitte, S., Joukoff, A., Frohlich, C., Laclare, F., Delmas, C., \& Boumier, P. 1999, AdSpR, 24, 205

Jablonski, F. 1991, private communication

Jilinski, E. G., Puliaev, S., Penna, J. L., Andrei, A. H. \& Laclare, F. 1999, A\&AS, 135, 227

Jilinski, E. G., Puliaev, S., Penna, J. L., Andrei, A. H., Sinceac, V., Chollet, F. \& Delmas, C. 1998, A\&AS, 130, 317

Laclare, F., Delmas, C., Coin, J. P. \& Irbah, A. 1996, SoPh, 166, 211

Puliaev, S., Penna, J. L., Jilinski, E. G. \& Andrei, A. H. 2000, A\&AS, 143, 265

Ribes, E., Beardsley, B., Brown, T.M., DeLache, Ph., Laclare, F. \& Leister, N.V. 1991 in The Sun in Time, ed. C.P. Sonett, M.S. Giampapa \& M.S. Mattews, 59

Rozelot, J. 1998, SoPh, 177, 321

Sinceac, V. 1998 in Analyse et exploitation des observations du Soleil à partir de l'astrolabe Danjon équipé d'une caméra $\mathrm{CCD}, \mathrm{PhD}$ thesis at Paris Observatory 